\title{
Potencial de generación de biogás y energía eléctrica Parte I: excretas de ganado bovino y porcino
}

\author{
Biogas and Power Generation Potential \\ Part I: Bovine and Pig Manure
}

\author{
Vera-Romero Iván \\ Universidad de la Ciénega del Estado de Michoacán de Ocampo \\ Correo.ivanverar@gmail.com \\ Martínez-Reyes José \\ Universidad de la Ciénega del Estado de Michoacán de Ocampo \\ Correo.jmreyes@geociencias.unam.mx
}

\author{
Estrada-Jaramillo Melitón \\ Universidad de la Ciénega del Estado de Michoacán de Ocampo \\ Correo:unoesun@yahoo.com \\ Ortiz-Soriano Agustina \\ Universidad de la Ciénega del Estado de Michoacán de Ocampo \\ Correo:ortizagus@yahoo.com.mx
}

Información del artículo: recibido: febrero de 2013, reevaluado: abril de 2013, aceptado: junio de 2013

\section{Resumen}

El trabajo reporta el potencial de energía a través del biogás obtenido directamente de las excretas del ganado vacuno y porcino con el que cuenta la región Ciénega correspondiente al Estado de Michoacán de Ocampo, México. Se empleó información del último censo agropecuario del INEGI para conocer la población de ganado, posteriormente, se estableció una cantidad de estiércol por tipo de animal y edad acorde a un tamaño promedio. Se calculó la cantidad de estiércol total y se estimó la cantidad de biogás que se podría obtener, así como la energía eléctrica. Representando un ahorro de energía eléctrica de $4.23 \%$ que corresponde para 2013 a un monto de $\$ 18,300,000$ pesos aproximadamente, con un costo promedio de 2.326 pesos por cada kWh en una tarifa 5A de la Comisión Federal de Electricidad (CFE).

\footnotetext{
Abstract

The potential energy through biogas obtained directly from the dung of bovine and pigs is reported in this paper, in the Cienega region of Michoacan, Mexico. The last INEGI Agricultural Census was used to determine the livestock population, and then the amount of manure by type of animal was established according to an average size. The total amount of manure was calculated and the amount of biogas and electricity produced. Representing a saving of electrical energy corresponding to $4.23 \%$ in 2013 to an amount of $\$ 18,300,00$ Mexican pesos approximately, with an average cost of 2.326 pesos per $\mathrm{kWh}$ at a rate $5 \mathrm{~A}$ Federal Electricity Commission (CFE).
}

\section{Descriptores:}

- biogas

- excretas

- ganado bovino y porcino

- energía eléctrica

\section{Keywords:}

- biogas

- dung

- bovine and pig cattle

- electric power 


\section{Introducción}

La región Ciénega de Michoacán de Ocampo ubicada al noroeste de esta entidad (figura 1), desde hace mucho tiempo es una zona principalmente agrícola y en menor medida pecuaria, hoy en día, esta práctica es uno de los pilares principales de su economía. Esta región está comprendida por nueve municipios: Briseñas, Cojumatlán de Régules, Jiquilpan, Pajacuarán, Sahuayo, Venustiano Carranza, Villamar, Vista Hermosa e Ixtlán. La característica principal que existe entre estos municipios es que todos están interconectados por carreteras que hacen relativamente fácil el acceso entre una y otra comunidad.

El principal ganado que destaca en esta región es el vacuno y porcino, con una existencia reportada por el Censo Agropecuario 2007, último realizado por el INEGI (www.inegi.org.mx), de 31,598 cabezas de ganado vacuno y 38,515 cabezas de porcino. Es probable que estas cifras a la fecha hayan variado, sin embargo, esa variación no debe ser significativa debido a que son actividades ligadas a su economía.

\section{Problemática}

La gran cantidad de estiércol producido por el ganado no tiene un tratamiento previo o adecuado para su disposición final, lo cual implica mantener un foco de infección latente perjudicial para la comunidad en general. Gran cantidad de estiércol se deposita en las tierras de cultivo que sirve para mejorar su calidad, así como para obtener mejores cosechas (Serrato et al., 2002), sin embargo, esta práctica tiene un inconveniente, el estiércol tarda aproximadamente un año en degra-

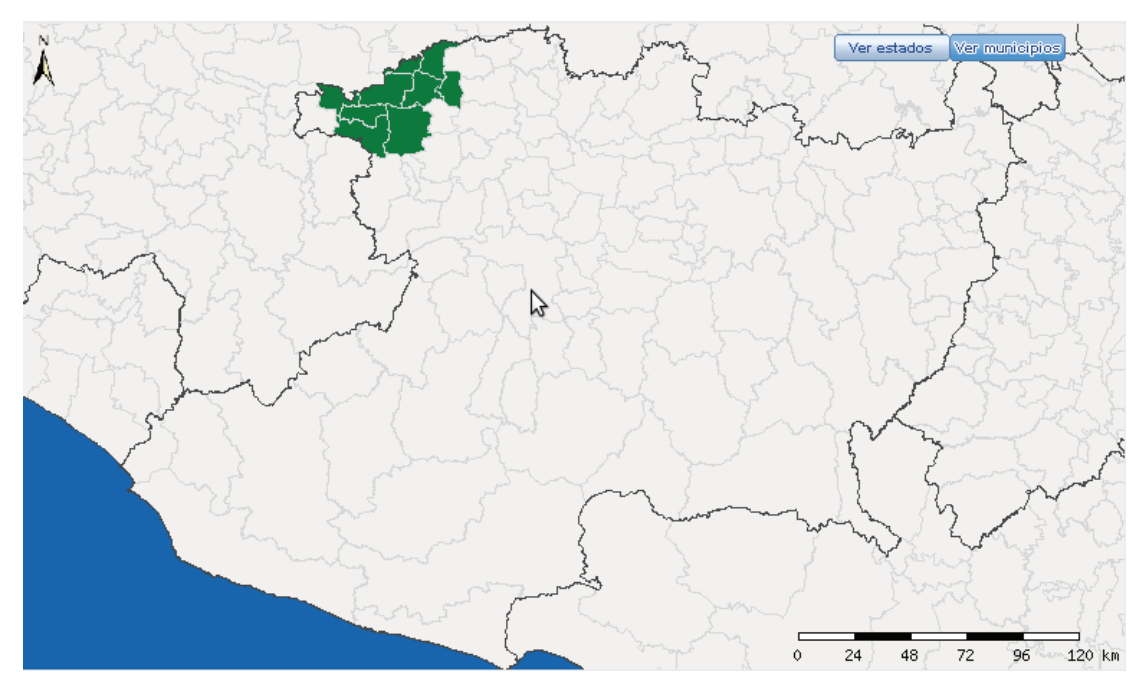

Figura 1. Región Ciénega. Fuente: INEGI darse y poder ofrecer todos sus nutrientes de manera asimilable (Herrick y Lai, 1996; Aarons et al., 2004; Vadas et al., 2011). Otra desventaja es que al descomponerse en presencia del aire la reacción que se presenta es exotérmica a través de una fermentación, el calor producido eleva la temperatura a su alrededor y es capaz de inhibir la germinación de la semilla provocando daño a las plántulas, lo cual es resultado de una relación incorrecta de tierra/estiércol.

Otra práctica de tratamiento final de las excretas es verterlas a canales, lo que se presenta principalmente en la zona de "La Palma", ya que en época de lluvias el estiércol no se puede recoger y es desalojado de esa forma. Una práctica más de disposición final es emplear lagunas de sedimentación, sin embargo no es una práctica muy común, por lo tanto no resuelve el problema.

Por otro lado, se tiene el problema de los altos costos y la escasez en los energéticos (BP, 2012), que se usan principalmente para la producción de energía eléctrica. Para el año 2009, la región Ciénega, consumió 186,601 MWh de un total de 5,928,038 MWh que consumió el Estado completo y que representó un monto aproximado de 230 millones de pesos (17 millones de USD), de acuerdo con los datos reportados por el INEGI. Empleando una paridad promedio del peso contra el dólar, basado en datos de julio de 2011 a julio de 2012, de 13.5 pesos por USD (www.banxico.org.mx).

En este trabajo se pretende estimar el potencial que tiene esta región para la producción de biogás a través de excretas de ganado vacuno y porcino, además de estimar la generación de energía que se podría obtener empleando este biocombustible como energético para producir energía eléctrica (Casas et al., 2009) que se aplicaría al alumbrado municipal de esta misma región.

\section{Desarrollo}

\section{Clasificación}

Para cuantificar la cantidad de biogás producido se recurrió a la base de datos del INEGI correspondiente al Censo Agropecuario 2007, donde se muestra la cantidad de cabezas de ganado dispuestas por edades, dependiendo el tipo de ganado. Para el estudio se consideraron tanto el ganado vacuno como el porcino, tomando en cuenta que la región Ciénega es una zona agropecuaria 
importante del Estado. Las edades para cada uno de los ganados corresponden a un tamaño como se puede apreciar en la tabla 1, con el fin de definir la cantidad de estiércol promedio producido por grupo (Martínez, 2007).

La cantidad de excretas producidas por cabeza varía dependiendo del tamaño, sin embargo, el tiempo de estación de los animales en el corral impacta directamente en la cantidad que se puede aprovechar de ellas (tabla 2). Para el ganado vacuno, el tiempo de estación es de doce horas por día aproximadamente, lo cual implica que solo $50 \%$ de las excretas se puede recuperar,

Tabla 1. Clasificación por edad

\begin{tabular}{ccc}
\hline & Menores de 1 año & Ternero \\
Vecuno 1 a 2 años & Pequeño \\
De 2 a 3 años & Mediano \\
Mayores de 3 años & Grande \\
\hline \multirow{2}{*}{ Porcino } & Menores de 8 semanas & Pequeño \\
& Entre 2 y 6 meses & Mediano \\
& Sementales & Grande \\
& Vientres & Grande \\
\hline
\end{tabular}

mientras que para el ganado porcino, el estadio es de $100 \%$, dando como resultado un eficaz método de recolección de excretas.

\section{Existencias y producción de estiércol}

La existencia de ganado se reporta por cada municipio que comprende la región Ciénega (tablas 3-4), mientras que la cantidad total de excretas dependerá de la cantidad total de cabezas de ganado afectado por su tamaño (tablas 5-7).

Tabla 2. Producción promedio de estiércol de acuerdo al tiempo de estación (Martínez, 2007)

\begin{tabular}{ccc}
\hline \multicolumn{3}{c}{ Bovino } \\
\hline \multicolumn{3}{c}{ Producción de excretas por día } \\
\hline Grande & 15 & $\mathrm{~kg} / \mathrm{d}$ ía \\
Mediano & 10 & $\mathrm{~kg} /$ día \\
Pequeño & 8 & $\mathrm{~kg} /$ día \\
Ternero & 4 & $\mathrm{~kg} /$ día \\
\hline \multicolumn{3}{c}{} \\
\hline Producción de excretas por día \\
\hline Grande & 2 & $\mathrm{~kg} /$ día \\
Mediano & 1.5 & $\mathrm{~kg} /$ día \\
Pequeño & 1 & $\mathrm{~kg} /$ día \\
\hline
\end{tabular}

Tabla 3. Cabezas de ganado vacuno por municipio. Fuente: Adaptación datos INEGI

\begin{tabular}{cccccc}
\hline Región Ciénega, Michoacán & Ternero & Pequeño & Mediano & Grande & TOTAL \\
\hline Briseñas & 271 & 265 & 336 & 113 & 985 \\
Cojumatlán de Régules & N/D & N/D & N/D & N/D & N/D \\
Jiquilpan & 2,492 & 1,851 & 1,261 & 2,467 & 8,071 \\
Pajacuarán & 793 & 556 & 556 & 818 & 2,723 \\
Sahuayo & 885 & 770 & 621 & 1,127 & 3,403 \\
Venustiano Carranza & 1,420 & 1,240 & 1,441 & 1,664 & 5,765 \\
Villamar & 920 & 867 & 722 & 1,360 & 3,869 \\
Vista Hermosa & 453 & 547 & 489 & 811 & 2,300 \\
Ixtlán & 837 & 549 & 572 & 1,082 & 3,040 \\
\hline Total & 8,071 & 6,645 & 5,998 & 9,442 & 30,156 \\
\hline Porcentaje (\%) & 26.8 & 21.0 & 19.9 & 31.3 & 100 \\
\hline
\end{tabular}


Tabla 4. Cabezas de ganado porcino por municipio. Fuente: Adaptación datos INEGI

\begin{tabular}{ccccc}
\hline Región Ciénega, Michoacán & Pequeño & Mediano & Grande & TOTAL \\
\hline Briseñas & 339 & 567 & 345 & 1,251 \\
Cojumatlán de Régules & $\mathrm{N} / \mathrm{D}$ & $\mathrm{N} / \mathrm{D}$ & $\mathrm{N} / \mathrm{D}$ & $\mathrm{N} / \mathrm{D}$ \\
Jiquilpan & 87 & 85 & 52 & 241 \\
Pajacuarán & 3,149 & 3,683 & 832 & 7,664 \\
Sahuayo & 21 & 44 & 14 & 79 \\
Venustiano Carranza & 112 & 495 & 215 & 822 \\
Villamar & 57 & 263 & 148 & 468 \\
Vista Hermosa & 7,461 & 14,069 & 2,626 & 24,156 \\
Ixtlán & 941 & 1,113 & 603 & 2,657 \\
\hline Total & 12,167 & 20,319 & 4,835 & 37,321 \\
\hline Porcentaje (\%) & 32.6 & 54.4 & 13.0 & 100 \\
\hline
\end{tabular}

Tabla 5. Producción de estiércol Bovino (kg/día)

\begin{tabular}{cccccc}
\hline Región Ciénega, Michoacán & Ternero & Pequeño & Mediano & Grande & TOTAL \\
\hline Briseñas & 1,084 & 2,120 & 3,360 & 1,695 & 8,259 \\
Cojumatlán de Régules & N/D & N/D & N/D & N/D & N/D \\
Jiquilpan & 9,968 & 14,808 & 12,610 & 37,005 & 74,391 \\
Pajacuarán & 3,172 & 4,448 & 5,560 & 12,270 & 25,450 \\
Sahuayo & 3,540 & 6,160 & 6,210 & 16,905 & 32,815 \\
Venustiano Carranza & 5,680 & 9,920 & 14,410 & 24,960 & 54,970 \\
Villamar & 3,680 & 6,936 & 7,220 & 20,400 & 38,236 \\
Vista Hermosa & 1,812 & 4,376 & 4,890 & 12,165 & 23,243 \\
Ixtlán & 3,348 & 4,392 & 5,720 & 16,230 & 29,690 \\
\hline Total & 32,284 & 53,160 & 59,980 & 141,630 & 287,054 \\
\hline Porcentajes (\%) & 11.2 & 18.5 & 20.9 & 49.3 & 100 \\
\hline
\end{tabular}

Tabla 6. Producción de estiércol Porcino (kg/día)

\begin{tabular}{ccccc}
\hline Región Ciénega, Michoacán & Pequeño & Mediano & Grande & TOTAL \\
\hline Briseñas & 339 & 850 & 690 & 1,879 \\
Cojumatlán de Régules & $\mathrm{N} / \mathrm{D}$ & $\mathrm{N} / \mathrm{D}$ & $\mathrm{N} / \mathrm{D}$ & $\mathrm{N} / \mathrm{D}$ \\
Jiquilpan & 87 & 127 & 104 & 318 \\
Pajacuarán & 3,149 & 5,524 & 1,664 & 10,337 \\
Sahuayo & 21 & 66 & 28 & 115 \\
Venustiano Carranza & 112 & 742 & 430 & 1,284 \\
Villamar & 57 & 394 & 296 & 747 \\
Vista Hermosa & 7,461 & 21,103 & 5,252 & 33,816 \\
Ixtlán & 941 & 1,669 & 1,206 & 3,816 \\
\hline Total & 12,167 & 30,479 & 9,670 & 52,316 \\
\hline Porcentajes (\%) & 23.3 & 58.3 & 18.5 & 100 \\
\hline
\end{tabular}

Tabla 7. Producción de estiércol total

\begin{tabular}{ccc}
\hline Tipo de ganado & $\mathrm{kg} /$ día & $\mathrm{Kg} / \mathrm{año}$ \\
\hline Vacuno & 287,054 & $104,774,710$ \\
Porcino & 52,316 & $19,095,158$ \\
\hline TOTAL & 339,370 & $123,869,868$ \\
\hline
\end{tabular}

\section{Producción de biogás}

El biogás se produjo a través de un proceso de descomposición anaeróbico de los estiércoles, pasando por las siguientes fases: hidrólisis por bacterias fermentativas, acidogénesis y acetogénesis, para finalmente formarse el metano en la etapa de metanogénesis (Temelis y Ulloa, 2007). La producción de biogás obedecerá al tamaño y especie del animal sin tomar en cuenta una temperatura promedio anual ni la eficiencia de reacción anaerobia intrínseca del proceso de manera directa. El biogás estimado se obtuvo con los valores de la tabla 8 , entre el estiércol producido por día de cada uno de los tamaños y especies estudiadas, afectado por el total de estiércol. La producción de biogás se observa en las tablas 9 y 10.

A pesar de que no se realiza un estudio de bonos de carbono a través del mecanismo de desarrollo limpio debido a que su primer periodo de compromiso culmina a finales de 2012 (Naciones Unidas, 1998), sí se pre- 
Tabla 8. Producción de biogás unitaria (Martínez, 2007)

\begin{tabular}{cc}
\hline Bovinos & Producción $\mathrm{m}^{3} /$ animal día \\
\hline Grande & 0.6 \\
Mediano & 0.4 \\
Pequeño & 0.32 \\
Ternero & 0.16 \\
\hline Cerdos & Producción $\mathrm{m}^{3} /$ animal día \\
\hline Grande & 0.14 \\
Mediano & 0.1 \\
Pequeño & 0.07 \\
\hline
\end{tabular}

Tabla 9. Producción de biogás en $\mathrm{m}^{3}$ /día por municipio y tamaño de ganado Bovino

\begin{tabular}{cccccc}
\hline Región Ciénega, Michoacán & Ternero & Pequeño & Mediano & Grande & TOTAL \\
\hline Briseñas & 43 & 85 & 134 & 68 & 330 \\
Cojumatlán de Régules & $\mathrm{N} / \mathrm{D}$ & $\mathrm{N} / \mathrm{D}$ & $\mathrm{N} / \mathrm{D}$ & $\mathrm{N} / \mathrm{D}$ & N/D \\
Jiquilpan & 399 & 592 & 504 & 1,480 & 2,976 \\
Pajacuarán & 127 & 178 & 222 & 491 & 1,018 \\
Sahuayo & 142 & 246 & 248 & 676 & 1,313 \\
Venustiano Carranza & 227 & 397 & 576 & 998 & 2,199 \\
Villamar & 147 & 277 & 289 & 816 & 1,529 \\
Vista Hermosa & 72 & 175 & 196 & 487 & 930 \\
Ixtlán & 134 & 176 & 229 & 649 & 1,188 \\
\hline Total & 1,291 & 2,126 & 2,399 & 5,665 & 11,482 \\
\hline Porcentajes (\%) & 11 & 19 & 21 & 49 & 100 \\
\hline
\end{tabular}

Tabla 10. Producción de biogás en $\mathrm{m}^{3}$ /día por municipio y tamaño de ganado Porcino

\begin{tabular}{ccccc}
\hline Región Ciénega, Michoacán & Pequeño & Mediano & Grande & TOTAL \\
\hline Briseñas & 24 & 60 & 48 & 132 \\
Cojumatlán de Régules & N/D & N/D & N/D & N/D \\
Jiquilpan & 6 & 9 & 7 & 22 \\
Pajacuarán & 220 & 387 & 116 & 724 \\
Sahuayo & 1 & 5 & 2 & 8 \\
Venustiano Carranza & 8 & 52 & 30 & 90 \\
Villamar & 4 & 28 & 21 & 52 \\
Vista Hermosa & 522 & 1,477 & 368 & 2,367 \\
Ixtlán & 66 & 117 & 84 & 267 \\
\hline Total & 852 & 2,133 & 677 & 3,662 \\
\hline Porcentajes (\%) & 23 & 58 & 18 & 100 \\
\hline
\end{tabular}

senta un estimado de $\mathrm{CO}_{2} \mathrm{eq}$, que se dejaría de arrojar a la atmósfera por el hecho de quemar el metano contenido en el biogás, considerando $50 \%$ en volumen de $\mathrm{CH}_{4}$ (tabla 11) y una vida útil del proyecto a 21 años.

La reducción estimada de emisiones anuales podría comercializarse o negociarse con algún país interesado mediante el mecanismo de desarrollo limpio (MDL) (Flores et al., 2008).

\section{Generación de energía eléctrica}

Para la estimación de la energía que contiene el biogás, se empleó la información que contiene el software del Modelo de Biogás Mexicano v.2.0 (LMOP, 2009), empleando un poder calorífico de $18,852 \mathrm{~kJ} / \mathrm{m}^{3}$ de biogás. La eficiencia de los motores de combustión interna que se utilizan para la generación de energía eléctrica a través de biogás (Jenbacher de GE Tipo 2), con potencia eléctrica de $335 \mathrm{~kW}$ es de $36.3 \%$ (ISO) (General Electric, 2013), sin embargo, esta última decreció debido a las condiciones de sitio. Dado que no se evaluó una simulación del motogenerador, se consideró un valor de la eficiencia de transformación de $30 \%$ como un dato conservador para no mostrar resultados muy elevados y que no pudieran alcanzarse en la realidad; con un factor de planta del 0.9. Al obtener una generación de biogás de $5.53 \mathrm{Mm}^{3}$ y 2.76 $\mathrm{Mm}^{3}$ de $\mathrm{CH}_{4}$, con una energía de biogás de 28,947 MWh y 7,884 MWh de energía eléctrica producida, todo permanece constante por año de vida útil del proyecto. Se debe tomar en cuenta que el primer año del proyecto aún no genera energía eléctrica, ya que está asignado a la parte de la construcción y puesta en marcha. 


\section{Discusión y análisis de resultados}

La energía eléctrica que se puede generar a través del biogás producido por excretas de ganado vacuno y porcino es capaz de sustituir $4.23 \%$ aproximadamente de todo el consumo anual de la región Ciénega, sin embargo, monetariamente se podrían ahorrar $\$ 12,309,306$ M.N. (911,800 USD) aproximadamente en el año 2013. Los montos de costos de energía eléctrica reportados en el INEGI 2010 corresponden al año 2009, para realizar la aproximación de ahorro al 2013, se afectó la cantidad en $6 \%$ anual de incremento en la factura (tabla 12). Mientras que el costo unitario promedio anual de la energía eléctrica resultó ser de 1.24\$/kWh en 2009, 1.31 \$/kWh en 2010, 1.39 \$/kWh en 2011, 1.47 \$/kWh en 2012 y para 2013 sería de $1.56 \$ / \mathrm{kWh}$.
Estos últimos promedios unitarios anuales contemplaron todo tipo de tarifas de la CFE (comercial, industria, servicios, entre otras), sin embargo, la tarifa para el alumbrado público municipal se encuentra en los servicios públicos, haciendo que este proyecto tenga mayores ventajas. La tarifa promedio anual $5 \mathrm{~A}$ de la CFE resulta tener un costo mayor que la reportada por el INEGI (tabla 13) (www.cfe.gob.mx).

El ahorro con estos últimos datos sugiere un aumento significativo, independientemente de la tensión que se trate (tabla 14), suponiendo un ahorro de más de $\$ 18,300,000$ pesos anuales $(\$ 1,358,198$ USD), promediando ambas tensiones $(50 \% / 50 \%)$. Este último ahorro corresponde al más indicado, ya que sería en la parte de alumbrado público donde tendría más rentabilidad un proyecto municipal con estas características.

Tabla 11. Reducción de emisiones estimada por vida útil del proyecto

\begin{tabular}{cc}
\hline 41,557 & $\mathrm{CH}_{4}$ \\
\hline $18,326,579$ & $\mathrm{CO}_{2} \mathrm{eq}$ \\
\hline
\end{tabular}

Tabla 12. Costo de energía eléctrica. Fuente: Adaptación datos INEGI

\begin{tabular}{lc}
\hline \multicolumn{1}{c}{ Población } & $\begin{array}{c}\text { Costo } \\
\$ / \text { año }\end{array}$ \\
\hline Briseñas & $8,753,000$ \\
Cojumatlán de Régules & $8,778,000$ \\
Jiquilpan & $46,225,000$ \\
Pajacuarán & $14,286,000$ \\
Sahuayo & $79,696,000$ \\
Venustiano Carranza & $19,360,000$ \\
Villamar & $13,520,000$ \\
Vista Hermosa & $30,516,000$ \\
Ixtlán & $9,635,000$ \\
Total 2009 & $230,769,000$ \\
2010 & $244,615,140$ \\
2011 & $259,292,048$ \\
2012 & $274,849,571$ \\
2013 & $291,340,546$ \\
\hline
\end{tabular}

Tabla 13. Costo promedio unitario de energía eléctrica anual (\$/kWh), tarifa 5A. Fuente: Adaptación CFE

\begin{tabular}{cccccc}
\hline & & & & & Calculado \\
\hline Tensión & 2009 & 2010 & 2011 & 2012 & 2013 \\
\hline Media & 1.686 & 1.785 & 1.892 & 2.003 & 2.124 \\
Baja & 2.004 & 2.124 & 2.251 & 2.385 & 2.528 \\
$\begin{array}{c}\text { Promedio } \\
50 \% / 50 \%\end{array}$ & 1.845 & 1.954 & 2.072 & 2.194 & 2.326 \\
\hline
\end{tabular}

Tabla 14. Ahorro en pesos Mexicanos, por concepto de energía eléctrica @ Tarifa 5A CFE

\begin{tabular}{cccccc}
\hline Tensión & 2009 & 2010 & 2011 & 2012 & 2013 \\
\hline Media & $13,292,424$ & $14,071,121$ & $14,916,528$ & $15,795,291$ & $16,743,008$ \\
Baja & $15,800,749$ & $16,746,222$ & $17,746,884$ & $18,800,308$ & $19,928,326$ \\
Promedio & $14,546,586$ & $15,408,672$ & $16,331,706$ & $17,297,799$ & $18,335,667$ \\
\hline
\end{tabular}


Gracias a que esta zona se encuentra interconectada, puede ubicarse un centro de acopio en donde se destinen las excretas hacia el Biodigestor (Mantilla et al., 2007). Otra alternativa puede ser la generación distribuida, localizando a los biodigestores y plantas generadoras de energía de manera estratégica para aprovechar la ya existente red de CFE (Huttunen, 2011).

El agua que se obtiene después de pasar por el Biodigestor puede ser bombeada a tierras de cultivo o directamente a un canal o cuerpo de agua cercano, mientras que el abono orgánico derivado de la degradación de las excretas se puede depositar directamente en los campos de cultivo de la misma región o en tierras de bajo rendimiento, al mismo tiempo que sustituye gran parte del consumo de fertilizantes comerciales de origen químico (Osorio et al., 2007).

Este proyecto se puede realizar a través de la cooperación de la iniciativa privada, involucrando a los ganaderos y fideicomisos nacionales e internacionales (SENER y GTZ, 2009).

A pesar de que el municipio de Cojumatlán de Régules no presenta datos sobre existencias de ganado, se consideró parte de la región Ciénega para que se tome en cuenta y se incentive la ganadería para contribuir con este proyecto.

\section{Conclusiones}

El aprovechamiento de las excretas de ganado bovino y porcino para la generación de biogás y obtención de energía eléctrica para abastecer la región Ciénega del Estado de Michoacán de Ocampo, resulta ser altamente atractivo en cuanto al ahorro que se puede obtener en la factura de CFE en una tarifa 5A para alumbrado público, con una aportación energética total de $4.23 \%$ del consumo total de esta región. Así también, se contribuiría a la mitigación de la generación de $\mathrm{CO}_{2 \mathrm{eq}}$ de acuerdo a las disposiciones internacionales para la mitigación del calentamiento global.

\section{Referencias}

Aarons S.R., O'Connor C.R., Gourley C.J.P. Dung Decomposition in Temperate Dairy Pastures I. Changes in Soil Chemical Properties. Australian Journal of Soil Research, volumen 42, 2004: 107-114.

BP. BP, Statistical Review of world Energy, junio de 2012, Londres.

Casas-Prieto M.A., Rivas-Lucero B.A., Soto-Zapata M., SegoviaLerma A., Morales-Morales H.A., Cuevas-González M.I., Keissling-Divison C.M. Estudio de factibilidad para la puesta en marcha de los digestores anaeróbicos en establos lecheros en la cuenca de delicias, Chih. Revista Mexicana de Agroquímicos, volumen 8 (número 24), enero-junio de 2009: 745-756.

Flores R., Muñoz-Ledo R., Flores B.B., Cano K.I. Estimación de la generación de energía eléctrica a partir de biomasa para proyectos del programa de mecanismos de desarrollo limpio. Revista Mexicana de Ingeniería Química, volumen 7 (número 1), abril 2008: 35-39.

General Electric. Jenbacher GE Tipo 2, Productos [en línea] [fecha de consulta: 28 de abril de 2013]. Disponible en: <http://site. ge-energy.com/prod_serv/products/recip_engines/es/type2. htm>

Herrick J.E., Lai R. Dung Decomposition and Pedoturbation in a Seasonally Dry Tropical Pasture. Biol Fertil Soils, volumen 23, 1996: 177-181.

Huttunen S. Embeddedness in Local Farm-Scal Bioenergy Production. Revista de Estudios sobre Despoblación y Desarrollo Rural, (número 11), octubre de 2011: 107-127.

LMOP, 2009. Modelo de biogás mexicano v.2.0. [Programa ordenador], Washington, DC, [Usado: 27 de junio 2012]. Disponible en: http://www.epa.gov/lmop/international/mexicano. html.

Mantilla-González J.M., Duque-Daza C.A., Galeano-Urueña C.H. Diseño y estudio económico preliminar de una planta productora de biogás utilizando residuos orgánicos de ganado vacuno. Revista Ingeniería e Investigación, volumen 27 (número 3), diciembre de 2007: 133-142.

Martínez C.C. Volumen de biodigestores. Energía y tú, (número 39), julio-septiembre de 2007 [en línea]. [Fecha de consulta: 2 de octubre de 2012]. Disponible en: http://www.cubasolar.cu/ biblioteca/energia/Energia39/HTML/articulo04.htm.

Naciones Unidas. Protocolo de Kyoto de la Convención Marco de las Naciones Unidas Sobre el Cambio Climático, 1998 [en línea] [Fecha de consulta: 7 de octubre de 2012] Disponible en: http://unfccc.int/portal_espanol/documentacion/lista_de_documentos/items/3339.php.

Osorio-Saraz J.A., Ciro-Velásquez H.J., González-Sánchez H. Evaluación de un sistema de biodigestión en serie para clima frio. Revista Facultad Nacional de Agronomía Medellín, volumen 60 (número 2), 2007: 4145-4162.

Secretaria de Energía (SENER) y Deutsche Gesellschaft Technische Zusammenarbeit (GTZ) GmbH. Energías renovables para el desarrollo sustentable en México, 2009 [en línea]. Disponible en: http://www.sener.gob.mx/portal/Default.aspx?id=1433.

Serrato-Sánchez R., Ortíz-Arellano A., Dimas-López J., BerúmenPadilla S. Aplicación de lavado y estiércol para recuperar suelos salinos en la comarca lagunera, México. Terra Latinoamericana, volumen 20 (número 3), julio-septiembre, 2002: 329-336.

Temelis N.J., Ulloa P.A. Methane Generation in Landfills. Renewable Energy, volumen 32, 2007: 1243-1257.

Vadas P.A., Aarons S.R., Butler D.M., Dougherty W.J. A New Model for Dung Decomposition and Phosphorus Transformations and Loss in Runoff. Soil Research, volumen 49, 2011: 367-375. 


\section{Este artículo se cita: \\ Citación estilo Chicago}

Vera-Romero, Iván, José Martínez-Reyes, Melitón Estrada-JaramiIlo, Agustina Ortiz-Soriano. Potencial de generación de biogás y energía eléctrica. Parte I: excretas de ganado bovino y porcino. Ingeniería Investigación y Tecnología, XV, 03 (2014): 429-436.

\section{Citación estilo ISO 690}

Vera-Romero I., Martínez-Reyes J., Estrada-Jaramillo M., OrtizSoriano A. Potencial de generación de biogás y energía eléctrica. Parte I: excretas de ganado bovino y porcino. Ingeniería Investigación y Tecnología, volumen XV (número 3), julio-septiembre 2014: 429-436.

\section{Semblanza de los autores}

Iván Vera-Romero. Realizó los estudios de Licenciatura en el Instituto Tecnológico de Orizaba (ITO), en donde obtuvo el grado de ingeniero mecánico en 2001. Asimismo el grado de maestro en energía, sistemas energéticos: procesos y uso eficiente de la energía por la Universidad Nacional Autónoma de México en 2011. Actualmente labora como profesor-investigador de la Universidad de la Ciénega del Estado de Michoacán de Ocampo.

José Martínez-Reyes. Realizó los estudios de licenciatura en el Instituto Politécnico Nacional (IPN), en donde obtuvo el grado de ingeniero químico industrial en 1990. También obtuvo el grado de maestro en tecnología avanzada por el Instituto Politécnico Nacional en 2007. Actualmente labora como profesor-investigador de la Universidad de la Ciénega del Estado de Michoacán de Ocampo.

Melitón Estrada-Jaramillo. Realizó los estudios de licenciatura en la Universidad Michoacana de San Nicolás de Hidalgo (UMSNH), en donde obtuvo el grado de ingeniero mecánico en 2002. Asimismo, el grado de maestro en ciencias en ingeniería mecánica en el área de termofluidos: sistemas de postcombustión para la disminución de contaminantes, por la Universidad Michoacana de San Nicolás de Hidalgo en 2004. Actualmente labora como profesor-investigador en la Universidad de la Ciénega del Estado de Michoacán de Ocampo y es coordinador de la trayectoria de ingeniería en energía.

Agustina Ortiz-Soriano. Realizó los estudios de licenciatura en filosofía en la Universidad Autónoma Metropolitana (UAM). Obtuvo el grado de maestra en ciencias de la educación por el Instituto Superior de Ciencias de la Educación del Estado de México en 2009 y el grado de maestra en humanidades (filosofía política) en la UAM en 2010. Actualmente labora como profesor-investigador de tiempo completo en la Universidad de la Ciénega del Estado de Michoacán de Ocampo. 